\title{
A challenging palliation via novel therapeutic approach for unusual presenting with gingival metastasis from renal cell carcinoma relapsed eight years after nephrectomy
}

\author{
Asım Armağan Aydın' ${ }^{1}$ Ahmet Şükrü Alparslan², Hülya Ayık ${ }^{3}$ Erkan Kayıkçığlu' ${ }^{1}$ Banu Öztürk ${ }^{1}$ \\ ${ }^{1}$ Department of Medical Oncology, Antalya Training and Research Hospital, Antalya, Turkey \\ ${ }^{2}$ Department of Interventional Radiology, Antalya Training and Research Hospital, Antalya, Turkey \\ ${ }^{3}$ Department of Gynaecologic Oncology, Akdeniz University School of Medicine, Antalya, Turkey
}

DOI: $10.18621 /$ eurj.348318

\begin{abstract}
Renal cell carcinoma (RCC) is the third most frequently seen primary source of metastatic lesions of the oral cavity. Still oral cavity metastasis is a rarely seen clinical manifestation during the course of RCC. In a patient with a known history of RCC, in the presence of newly emerged oral and maxillofacial lesion(s), possibility of metastasis should be always thought of and pathological investigation should be performed as soon as possible. In RCC, metastatic involvement of oral cavity is generally accompanied by involvement of other organs with resultant lower chance of cure and poor prognosis. Especially in conditions which adversely affect functions of chewing and swallowing consequently nutrition and quality of life, surgery is contraindicated and local interventional methods transarterial chemoembolization, microwave ablation and radiofrequency ablation can be good palliative treatment alternatives. In advanced stages of the diseases with systemic involvement application of local interventional methods either solely or in combination with radiotherapy and surgery increases compliance to the systemic treatment and can contribute markedly to the prolongation of survival. Herein we aimed to present a case with unusual histopathological characteristics and presentation in whom we achieved a longer period of disease-free survival using a different local treatment method.
\end{abstract}

Keywords: Renal cell carcinoma; gingiva, metastasis; transarterial chemoembolization, microwave ablation

Received: November 1, 2017; Accepted: March 31, 2017; Published Online: April 1, 2018

$\mathbf{M}$ alignant tumors rarely metastasize to soft tissue of oral cavity and constitute only $1 \%$ of all malignancies of the oral region [1]. More than $80 \%$ of metastatic tumors arise from jaw bone and especially from its premolar and molar regions. However predominantly, soft tissue of gingiva (54\%) and tongue (33\%) are involved [1-4]. In most of the patients, previous diagnosis of primary tumor is already known, however oral cavity metastasis can encounter us as the first sign in $22-25 \%$ of the patients
[1]. Based retrospective data, as a primary source of oral cavity metastases, RCC ranks third after lung and breast cancers [1]. In $15 \%$ of the cases with disseminated renal cell carcinoma (RCC), oral cavity metastases can be seen and in most of the patients' synchronous involvement of other body regions can be observed. Clinical manifestations resemble to those detected in frequently seen inflammatory reactive and non-malignant systemic diseases of the oral cavity [4]. In differential diagnosis, in addition to

Address for correspondence: Asım Armağan Aydın, MD., Antalya Training and Research Hospital, Department of Medical Oncology, Meltem Boulevard, Muratpaşa, Antalya, Turkey 
histopathological examination, immunohistochemical staining is a very useful guiding tool in the characterization of primary tumor. Herein, we presented a patient who had undergone curative treatment with initial diagnosis of a local disease and applied to our clinic with symptoms of gingival involvement and concomitant systemic metastatic disease long after the establishment of the diagnosis. In this case we encountered difficulties in the differential diagnosis and especially treatment and thanks to alternative interventional methods of palliation his systemic treatment could be maintained, his quality of life, vital functions and survival could be improved. Under currently updated data, because of histopathological characteristics of the tumor (papillary type), its being an atypical metastasis of $\mathrm{RCC}$ and difference in the treatment applied, this is the first shared case in the literature. We aimed to present a unique case of RCC presented with a gingival metastasis treated with a novel local treatment.

\section{CASE PRESENTATION}

A 72-year-old male patient had undergone left radical nephrectomy 8 years ago with the diagnosis of papillary type RCC. His medical history revealed presence of hypertension and smoking. Because of early stage disease (T2N0M0) his clinical follow-ups were performed initially at 6 month, then yearly intervals with recommendations of NCCN. The patient presented to a private dental clinic with a broken tooth and subsequent edema and started to receive IV antibiotherapy with the diagnosis of periodontal infection. Partial treatment response was achieved and the patient applied to our oncology clinic with complaints of a mass on the right side of the mandibular, which gradually increased in size and caused severe pain, bleeding and swelling within the last one month. On his physical examination a hemorrhagic, erythematous and edematous mass lesion on the right mandibular region which restricted mandibular movements and measuring nearly 3-4 cm was detected (Figure 1A). Facial magnetic resonance imaging (MRI) revealed a destructive lesion on the right half of the mandibula. For staging purposes, 18F FDG PET/CT was performed which detected a hypermetabolic soft tissue lesion which caused destruction of the right lateral side of the mandibula (Suv max 17.8) and lesions consistent with metastatic involvement of mediastinum, right adrenal gland and L1, L2 and right iliac bone (Figures $1 \mathrm{~B}$ and 1C).

Biopsy material obtained from the right mandibular gingiva revealed findings consistent with RCC. In immunohistochemical examination also vimentin, EMA and CD-10 positivity and S-100 and CEA negativity were observed. In addition to RCC, initial diagnosis of odontogenic carcinoma and clear cell tumor of salivary gland was considered. Pathology preparations of the specimens obtained during the surgery performed 8 years ago were comparatively reevaluated and diagnosis of metastasis of papillary type RCC was confirmed (Figures 2A, 2B and 2C). The patient did not accept surgical treatment so palliative radiotherapy was initiated for the treatment of pain and chewing functions of the patient. However, on the 7th day of the treatment, no response to the
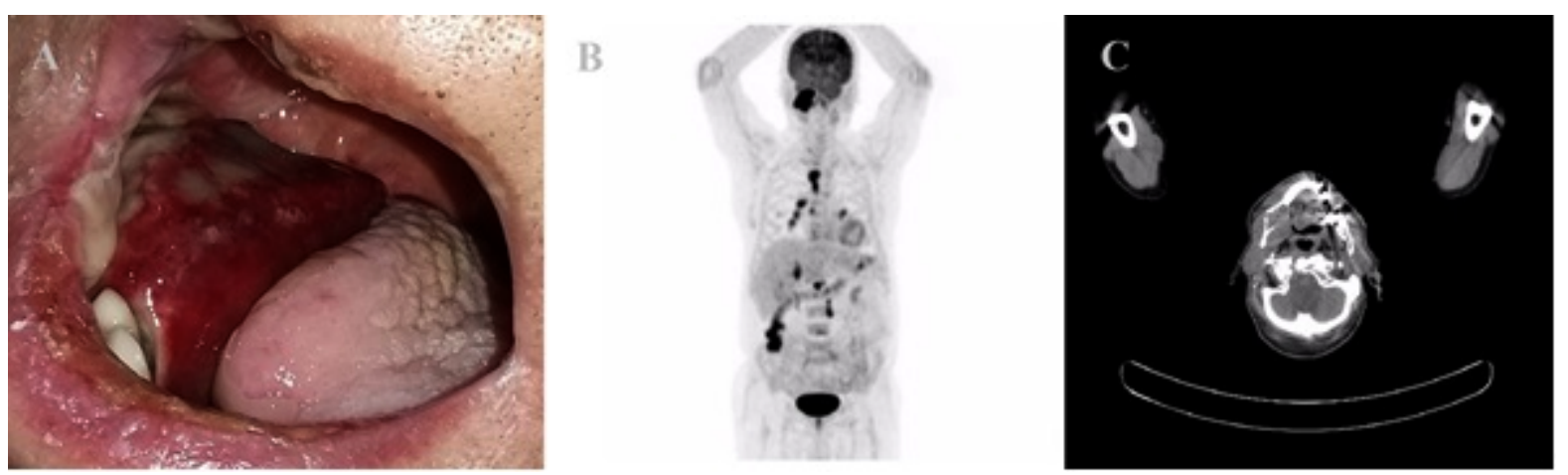

Figure 1. (A) A lesion on the right mandibular region nearly $4 \mathrm{~cm}$ in size surrounded by edematous and hemorrhagic tissue, (B and C) hypermetabolic soft tissue lesion which caused destruction of the bone tissue on 18-F FDG PET-CT. 

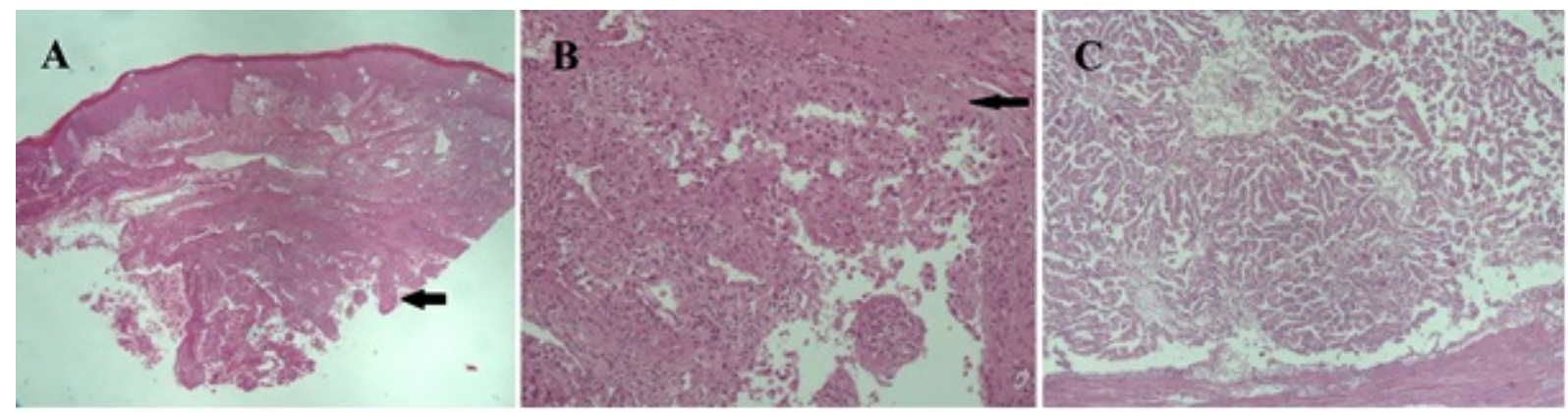

Figure 2. (A) Submucosal metastasis of malignant epithelial tumor (H\&E $\times 20)$, (B) Malignant papillary cells with eosinophilic cytoplasm examined under high magnification $(\mathrm{H} \& \mathrm{E} \times 100)$, (C) Renal cell carcinoma of papillary type $(\mathrm{H} \& \mathrm{E} \times 40)$.

therapy was taken, cause of increase in the size of the mass and intractable bleeding necessitated termination of radiotherapy. Interventional radiologist performed transarterial chemo-embolization (TACE) and microwave ablation (MA) for the mass perfused via temporal superficial artery. A catheter was inserted through femoral artery into the right external carotid artery to perform selective angiography. On angiograms two arteries supplying the right buccal mass (mandibular and angular arteries) were detected. Both of these arteries were embolized with Glue delivered via transarterial route. Since lesion on the right buccal region was a bilobar mass, one entry site was used for MA and 60 watt was delivered to both of these sites for 2 and 3 minutes (Figures 3A, 3B and $3 \mathrm{C})$. Marked tumoral shrinkage was observed after early post-TACE and post-MA period in the patient whose bleeding problem ceased and chewing function improved. Palliative treatment was restarted and completed. At the same time administration of thyrosine kinase inhibitor (sunitinib maleate) was initiated for the treatment of systemic disease. At present, at the first year of the treatment, the patient's condition is stable, and he is leading a healthy life.

\section{DISCUSSION}

RCC constitutes $2 \%$ of all malignancies in the whole world and every year. RCC has been associated with 300,000 new cases and 100,000 cases of death [5]. The most important risk factors associated with RCC are smoking, obesity and hypertension. Major histological variants include in order of decreasing incidence rates are clear cell (75-80\%), papillary (10$15 \%)$, chromophobe $(5 \%)$, sarcomatoid tumors $(5 \%)$ and tumors of the collecting tubuli (1\%). In patients who are initially diagnosed as local disease, rates of local recurrence and distant metastases are 2-5\% and $20-35 \%$, respectively. In patients who have synchronous distant metastases at the time of diagnosis, survival rates are lower than those
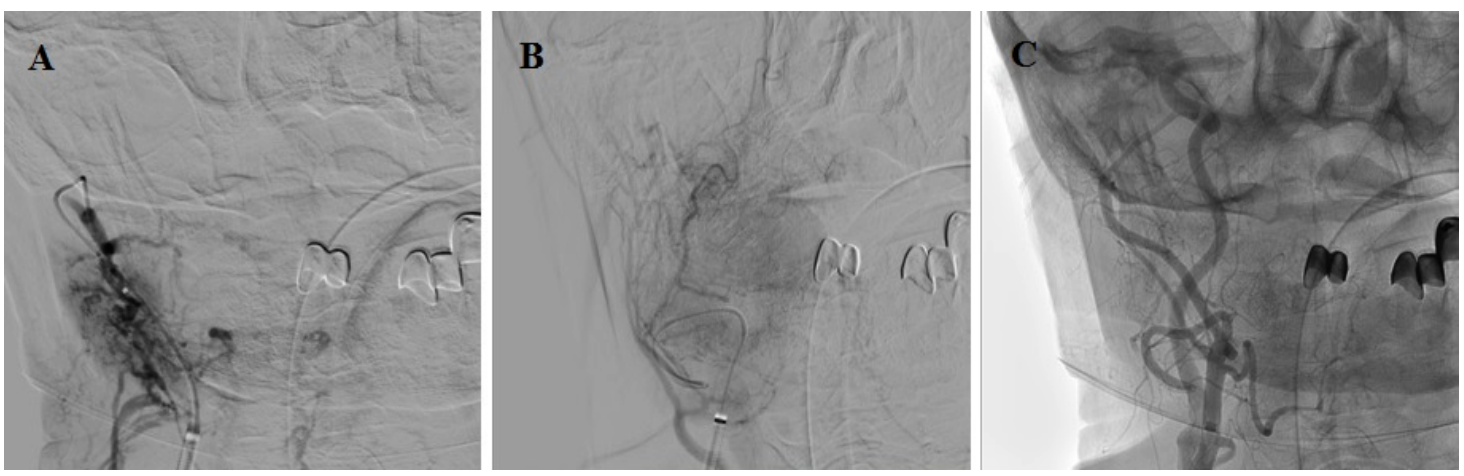

Figure 3. Selective angiographic images (A and B), Image of the lesion following microwave ablation procedure (C). 
developed metastases after nephrectomy. In our case, following curative treatment of local disease, systemic relapse emerged 8 years later and especially presentation of the first symptom concurrently with gingival involvement was a surprising and unexpected condition. In most of the patients, distant metastases are seen on multiple regions without any chance of curative intervention $[4,6]$. Still survival rates and quality of life of the patients who can undergo cytoreductive surgery and metastasectomy are more favorable. The most frequently seen regions of distant metastases are lungs, bone, liver, renal fossa and brain, however metastatic lesions can be seen in any organ. RCC very rarely metastasize into head and neck region and generally scarce number of case reports have been cited in the literature [7-13]. In local disease risk of metastasis into head and neck region is only $1 \%$, while in disseminated disease it rises up to $15 \%$. Tumor metastases into head and neck region are explained by retrograde flow through venous channels by way of prevertebral and vertebral venous plexuses because of increase in intraabdominal and intrathoracic pressures. Through this pathway RCC can metastasize through hematogenous route into head and neck region by bypassing pulmonary capillary circulation [14]. An unexpected clinical manifestation emerging on an atypical region during the course of $\mathrm{RCC}$ in a patient whose diagnosis is known, should always suggest the possibility of metastasis. Gingival inflammation may also facilitate settlement of the metastatic cells on gingiva. Therefore, oral hygiene is important.

Metastasis of solid tumors is a very rarely seen condition and constitute $1 \%$ of all oral cavity tumors. Still even though tumors metastasizing into jaw cannot be detected radiologically, especially in autopsy studies its incidence may be conceivably higher $(16 \%)$ than expected. In men most frequently seen primary sources are lungs, kidney, liver and prostate gland, while in women primary sources are mostly breast, genital organs, kidney, and colorectal region [15]. However, metastatic tumors of the oral cavity are more frequently seen at a somewhat earlier age in women, when compared with men (women; 5. decade, and men, 7. decade). Though jaw and especially mandibula are most frequently affected regions, gingiva leads the way in soft tissue involvement.

Clinical presentations in metastatic tumors of oral cavity can be observed in a wide spectrum including jaw pain, ulcerated exophytic lesion, lassitude, impaired chewing and swallowing functions and tooth loss. Similar symptoms can be seen in odontogenic infections, pyogenic granulomas, trauma, benign odontogenic tumors or they can manifest as symptoms of neurologic involvement as amyloidosis, sarcoidosis, multiple sclerosis or a non-metastatic disease. Because of extreme rarity of metastatic tumors of oral cavity and patients' presentation with subtle dental symptoms, especially clinicians should be very attentive in making differential diagnosis $[15,16]$. As is the case with our case, findings as faster growth and bone tissue destruction are strong indicators of metastatic disease. In a patient with a known history of RCC, newly emerging oral and maxillo-facial lesion should always suggest the presence of metastases and especially in patients with a known history of primary tumor, histopathological examination should be performed as soon as possible.

Although a routine algorithm concerning imaging techniques has not been formulated, in this case in the evaluation of local involvement and systemic disease, MRI and 18-F FDG PET-CT were used successively. Although histopathological examination is the gold standard in the differential diagnosis of primary tumor in suspect cases, immunohistochemical examination plays a guiding role in difficult cases similar to ours. Pax-8, CD-10 and vimentin positivity reinforces diagnosis of RCC. In our case, surgical material of the previously performed nephrectomy was compared histopathologically with gingival biopsy material and vimentin, EMA and CD-10 positivity was very helpful for definitive confirmation of the diagnosis.

Metastatic involvement of oral cavity is generally an indicator of end-stage disease. Most frequently, metastatic lesions in many other regions are present. Prognosis is unfavorable and treatment does not go beyond palliation in the majority of patients. In the literature mean survival time after oral cavity metastases has been reported as less than 1 year (range, 3.7-8.25 months) [1, 6]. In this case the reasons of survival times longer than anticipated include correction of vital functions as chewing and swallowing using local vascular interventional methods, completion of radiotherapy, improvement of quality of life, performance and nutritional status of the patient together with effective application of 
systemic treatment. In the light of current information, surgery is the standard treatment of isolated gingival metastasis of RCC. Limited number of literature data concerning use of local non-surgical methods in cases with metastatic lesions of head and neck region are available. However as is seen in this case, in patients whose clinical status is not amenable to surgery, those with systemic organ involvement or in patients who reject surgical treatment, in specialized centers radiotherapy as monotherapy or in combination with local interventional methods can be safely and reliably used so as to achieve improved palpation and quality of life and also increase patient compliance with resultant favorable contribution to his/her survival.

\section{CONCLUSION}

The right mandibular gingival lesion biopsy revealed findings consistent with RCC. Local interventional methods, transarterial chemoembolization, microwave ablation were done. At the first year of the treatment, the patient's condition is stable, and he is leading a healthy life. This approach may aid in the improvement of complications and vital functions and also it may exert favorable effects facilitating administration of palliative treatment modalities as radiotherapy. We think that by this means, compliance to systemic treatment may contribute substantially to the prolongation of survival.

\section{Informed consent}

Written informed consent was obtained from the patient for the publication of this case report.

\section{Conflict of interest}

The authors declared that there are no potential conflicts of interest with respect to the research, authorship, and/or publication of this article.

\section{REFERENCES}

[1] Hirshberg A, Shnaiderman-Shapiro A, Kaplan I, Berger R. Metastatic tumours to the oral cavity pathogenesis and analysis of 673 cases. Oral Oncol 2008;44:743-52.

[2] Servato JP, de Paulo LF, de Faria PR, Cardoso SV, Loyola AM. Metastatic tumours to the head and neck: retrospective analysis from a Brazilian tertiary referral centre. Int $J$ Oral Maxillofac Surg 2013;42:1391-6.

[3] McClure SA, Movahed R, Salama A, Ord RA. Maxillofacial metastases: a retrospective review of one institution's 15-year experience. J Oral Maxillofac Surg 2013;71:178-88.

[4] Owosho AA, Xu B, Kadempour A, Yom SK, Randazzo J, Ghossein RA, et al. Metastatic solid tumors to the jaw and oral soft tissue: A retrospective clinical analysis of 44 patients from a single institution. J Craniomaxillofac Surg 2016;44:1047-53.

[5] Ferlay J, Shin HR, Bray F, Forman D, Mathers C, Parkin DM. Estimates of worldwide burden of cancer in 2008: GLOBOCAN 2008. Int J Cancer 2010;127:2893-917.

[6] Van der Waal RI, Buter J, van der Waal I. Oral metastases: report of 24 cases. Br J Oral Maxillofac Surg 2003;41:3-6.

[7] Jatti D, Puri G, Aravinda K, Dheer DS. An atypical metastasis of renal clear cell carcinoma to the upper lip: a case report. J Oral Maxillofac Surg 2015; 3:371.e1-6.

[8] Narea-Matamala G, Fernandez-Toro Mde L, Villalabeitia-Ugarte E, Landaeta-Mendoza M, Rojas-Alcayaga G. Oral metastasis of renal cell carcinoma, presentation of a case. Med Oral Patol Oral Cir Bucal 2008;13:E742-4.

[9] Abbaszadeh-Bidokhty H, Motallebnejad M, Rajabi-Moghaddam M. Metastatic renal cell carcinoma presenting as a clear-cell tumor in tongue: a case report. Iran J Otorhinolaryngol 2014;26:185-90.

[10] Yoshitomi I, Kawasaki G, Mizuno A. Nishikido M, Hayashi T, Fujita S, et al. Lingual metastasis as an initial presentation of renal cell carcinoma. Med Oncol 2011;28:1389-94.

[11] Ahmadnia H, Amirmajdi NM, Mansourian E. Renal cell carcinoma presenting as mandibular metastasis. Saudi J Kidney Dis Transpl 2013;24:789-92.

[12] Ray A, Bhattacharya J, Ganguly S. Renal cell carcinoma presenting with oral tongue metastasis: a rare case presentation. J Cancer Res Ther 2013;9:117-8.

[13] Selvi F, Faquin WC, Michaelson MD, August M. Three synchronous atypical metastases of clear cell renal carcinoma to the maxillary gingiva, scalp and the distal phalanx of the fifth digit: a case report. J Oral Maxillofac Surg 2016;74:1286-e1-9.

[14] Bianchi M, Sun M, Jeldres C, Shariat SF, Trinh QD, Briganti A, et al. Distribution of metastatic sites in renal cell carcinoma: a populationbased analysis. Ann Oncol 2012;23:973-80.

[15] Hirshberg A, Berger R, Allon I, Kaplan I. Metastatic tumors to the jaws and mouth. Head Neck Pathol 2014;8:463-74.

[16] D'Silva NJ, Summerlin DJ, Cordell KG, Abdelsayed RA, Tomich CE, Hanks CT, et al. Metastatic tumors in the jaws: a retrospective study of 114 cases. J Am Dent Assoc 2006;137:1667-72. 